\title{
SHOCK WAVE PROPAGATION IN POROUS ICE
}

\author{
Sarah T. Stewart and Thomas J. Ahrens \\ Division of Geological and Planetary Sciences, California Institute of Technology, Pasadena, CA 91125
}

\begin{abstract}
We present data on shock wave propagation in porous ice under conditions applicable to the outer solar system. The equation of state of porous ice under low temperature and low pressure conditions agrees well with measurements under terrestrial conditions implying that data on terrestrial snow may be applicable to the outer solar system. We also observe rarefaction waves from small regions of increased porosity and calculate release wave velocities.
\end{abstract}

\section{INTRODUCTION}

Porosity effects are significant in impact processes, affecting the strength, elastic moduli and shock attenuation. Interest in the effects of porosity on collisional processes in the solar system has increased with the discoveries of the Kuiper Belt and asteroid Mathilde. Main belt asteroid 253 Mathilde has a bulk density of only $1.3 \mathrm{~g} / \mathrm{cm}^{3}$ (1) implying a bulk porosity greater than $50 \%$ (2). Mathilde's surface has craters with unusual morphologies whose formation may be linked to the high porosity of the object (3).

The Kuiper Belt is a disk of small icy bodies $(\mathrm{D} \leq 300 \mathrm{~km})$ similar to the Asteroid Belt but located outside the orbit of Neptune (4). At present, mutual collisions between Kuiper Belt Objects are erosional and destructive, producing dust and grinding the system into smaller bodies. The Kuiper Belt is the source of short period comets, and collisional evolution studies indicate that most comet-sized bodies are debris from collisions with larger bodies (4). The bulk porosity of comets has never been measured directly, but the Rosetta mission will measure both the bulk and surface porosity and other physical properties of a short period comet.

We have studied catastrophic disruption of porous materials (6) and we are also conducting experiments on shock wave propagation in porous ice and ice-dust mixtures. Here we present data on shock propagation in porous ice under conditions applicable to the outer solar system.

\section{EXPERIMENTS}

The temperatures of interest in the outer solar system are between about $80-150 \mathrm{~K}$. The velocities of mutual collisions between Kuiper Belt Objects are currently centered around $800 \mathrm{~m} / \mathrm{s}$ (5), producing peak pressures in the high hundreds of MPa. The porosity of comets is expected to range from $30-80 \%$. This is a large, and largely unexplored, range of states for shock studies on ice as most of the previous work on porous icc and natural snow have been conducted under low density $\left(<0.5 \mathrm{~g} / \mathrm{cm}^{3}\right)$, much lower $(<200 \mathrm{MPa})$ or much higher $(>3 \mathrm{GPa})$ pressures, or at warmer temperatures $\left(>-30^{\circ} \mathrm{C}\right)$.

Targets were prepared in the Caltech cold laboratory at a temperature of $-8^{\circ} \mathrm{C}$. Commercial crushed ice was ground and sifted to grain sizes between $180-355 \mu \mathrm{m}$ and hand pressed to the desired thickness and density. Discs were prepared ranging in thickness from $3-15 \mathrm{~mm}$ and densities between $0.3-0.6 \mathrm{~g} / \mathrm{cm}^{3}$.

Shock wave profiles were measured with 0.5 mil copper film electromagnetic velocity gauges insulated with 1 mil Kapton on each side (Dynasen, Inc., Goleta, CA). For shock wave studies in ice, velocity gauges are preferable over pressure gauges because they do not have to be calibrated for low temperatures. Because of the high porosity of the target, the gauges were custom made to a length of $9 \mathrm{~mm}$ and width of $4 \mathrm{~mm}$. Thus, the measurement is an average of the particle velocity over this area. The thin gauges equilibrate 


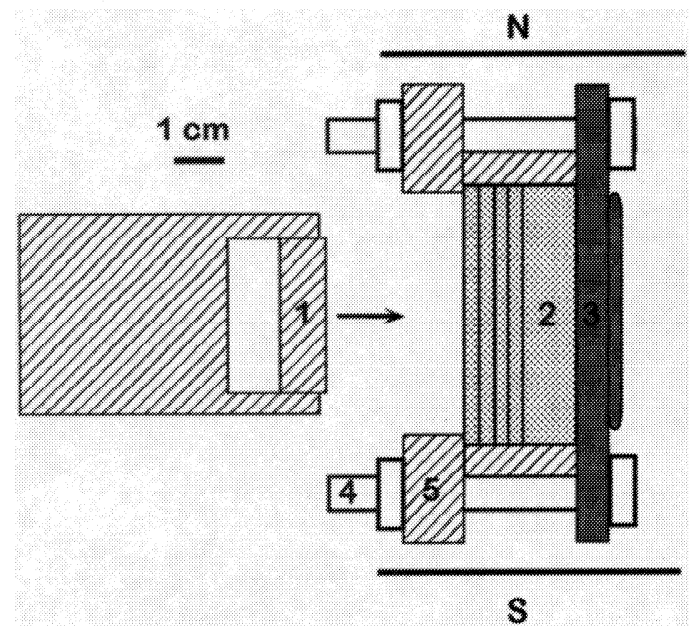

Figure 1: Experimental configuration cross section. Lexan flyer plate (1) in a Lexan sabot with an air gap behind the flyer. Porous ice discs (2) stacked in a Lexan cylinder with velocity gauges between each disc. The ice is cooled by conduction with a copper plate and cooling coil (3). Nylon screws and nuts (4) bind a front Lexan plate (5) to the copper plate, holding the ice discs in place. Thermocouples at the front and back of the ice monitor the temperature.

quickly and do not interfere with the rise of the shock wave.

The ice discs were stacked between two Lexan half-cylinders with velocity gauges centered between each disc. The system was cooled from the rear of the target by liquid nitrogen circulating through a copper coil braised to a copper plate. The shock wave does not reach the copper plate during the time period of interest. The experimental setup is shown in Fig. 1.

Impact experiments were conducted on the 40 $\mathrm{mm}$ gun in the Caltech Lindhurst Laboratory of Experimental Geophysics. We present data from shot \#1038, a supported wave under uniaxial strain, from an impact velocity of $1.05 \mathrm{~km} / \mathrm{s}$. The impactor was an $8 \mathrm{~mm}$-thick Lexan flyer plate with a diameter of $32 \mathrm{~mm}$. The target was porous ice at a density of $0.55 \mathrm{~g} / \mathrm{cm}^{3}$ and temperature of $\sim 100 \mathrm{~K}$. The target consisted of four $3 \mathrm{~mm}$-thick and one $10 \mathrm{~mm}$-thick ice discs $(\mathrm{D}=50.8 \mathrm{~mm})$ with four velocity gauges at $3,6,9$, and $12 \mathrm{~mm}$ from the target plane. The $10 \mathrm{~mm}$-thick disc distances

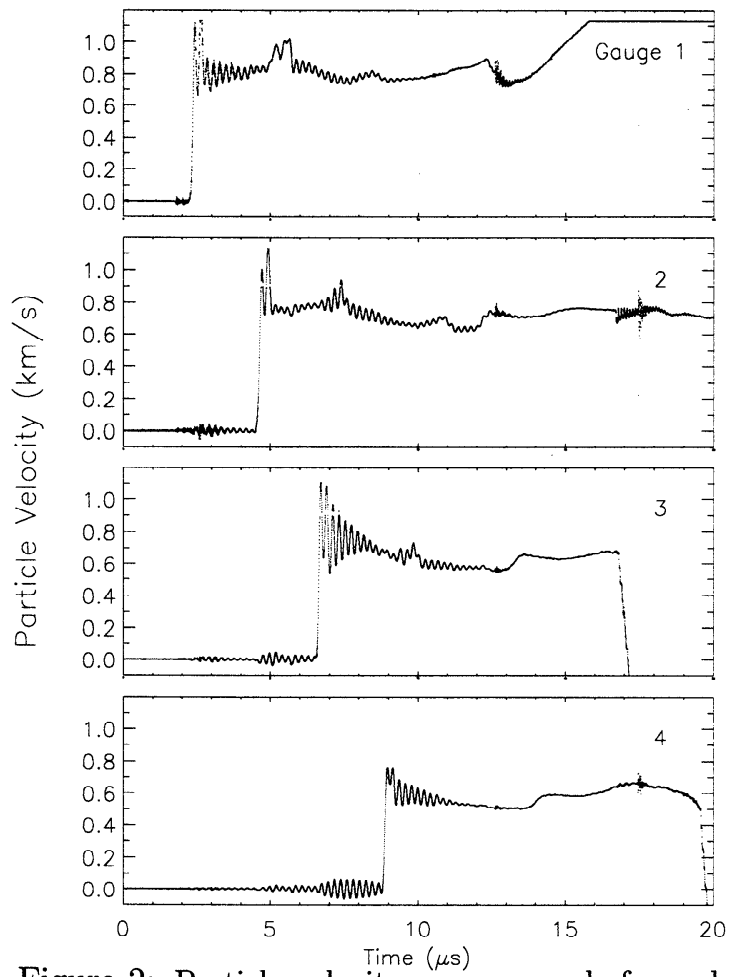

Figure 2: Particle velocity gauge records from shot 1038.

the active area of the experiment from the copper plate.

The target was hung in a constant uniform magnetic field ( 0.0865 Tesla). The voltage induced by the motion of the gauges is given by Faraday's Law, $E=B l v$, where $B$ is the strength of the magnetic field, $l$ is the length of the gauge and $v$ is the velocity of the gauge. The voltage was recorded on digital oscilloscopes at a time resolution of $2 \mathrm{~ns}$ and voltage resolution of 0.3 $\mathrm{mV}$. The systematic errors in the calculation of particle velocities are within $11 \%$.

\section{RESULTS}

The particle velocity data are shown in Fig. 2 . The raw data are noisy and show a $\sim 200$ ns oscillation on top of the velocity data from noise in the cables to the oscilloscopes. In this experiment, the time of impact was calculated based on the shock wave velocity between the embedded gauges and the thickness of the first ice disc.

From the shock wave arrival times at each gauge, the shock wave velocity is $1.31 \pm .03 \mathrm{~km} / \mathrm{s}$. 
The mean particle velocity at the peak of the shock wave, ignoring the initial spikes, are about $800 \mathrm{~m} / \mathrm{s}$ for the first guage, $760 \mathrm{~m} / \mathrm{s}$ and $740 \mathrm{~m} / \mathrm{s}$ for the second and third gauges, respectively. The fourth gauge peaks at 756 and levels off at about $580 \mathrm{~m} / \mathrm{s}$. The noise oscillation amplitude ranges from $20-200 \mathrm{~m} / \mathrm{s}$ and these peak particle velocities are mean values during the steady part of the shock wave. From these data, we calculate that the peak particle velocity was $760 \pm 40 \mathrm{~m} / \mathrm{s}$. Our values of $u_{s}$ and $u_{p}$ agree well with Furnish and Boslough's (7) equation of state data on natural snow at temperatures $>-36^{\circ} \mathrm{C}$. The linear equation of state, from least squares fit line through the combined data, is $c_{0}=-92 \mathrm{~m} / \mathrm{s}$ and $S=1.95$, for $390<u_{p}<1929 \mathrm{~m} / \mathrm{s}$.

From Lagrangian analysis (8) we derive the pressure vs. density curve for the rise of the shock wave, shown in Fig. 3. For the peak particle velocity, $760 \mathrm{~m} / \mathrm{s}$, the peak pressure from the shock wave is $0.587 \mathrm{GPa}$ and compressed density is 1.22 $\mathrm{g} / \mathrm{cm}^{3}$. Also shown is the theoretical calculation of the Hugoniot for $0.55 \mathrm{~g} / \mathrm{cm}^{3}$ ice from Bakanova et al. (9). The loading path intercepts the Hugoniot. Points $\mathrm{A}$ and $\mathrm{B}$ show the $3 \sigma$ upper and lower limits to the calibration of the particle velocity, comparable to the scatter of published equation of state data (7).

\section{DISCUSSION}

Our experiments under uniaxial strain have shown that we may accurately measure the equation of state of porous ice using Lagrangian electromagnetic velocity gauges. In addition there are several interesting features in the velocity gauge profiles which we explain herc.

First, when the shock wave arrives at each gauge there is an overshoot of roughly equal duration in each gauge before the particle velocity settles at the equation of state corresponding to the shock wave velocity. There are also repeated increases in particle velocity of finite duration in the first three gauges, labelled G\#a,b in Fig. 4.

If the interface between each ice disc contains a finite region of increased porosity (decreased density), the velocity gauges would exhibit a finite spike at the arrival of the shock wave due to the increased particle velocity for the equation of state of this region. The lower impedence in this

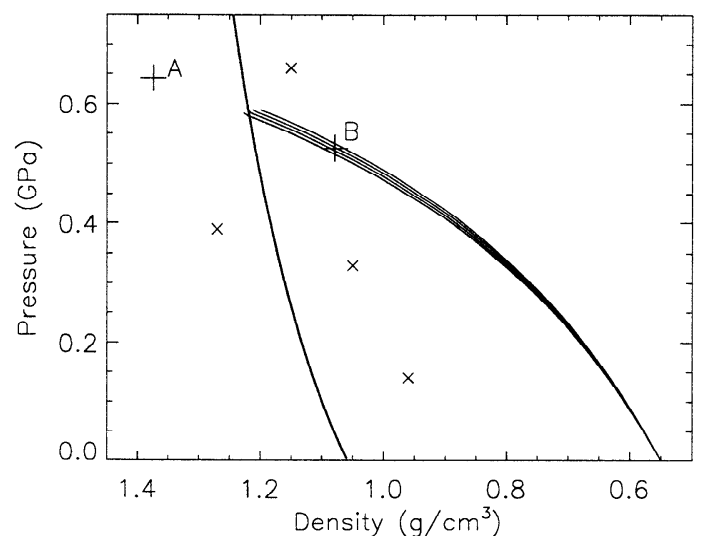

Figure 3: Shock wave stress vs. density loading paths derived from Lagrangian analysis of velocity gauges. The paths intercept the Hugoniot of $0.55 \mathrm{~g} / \mathrm{cm}^{3}$ ice (9) Published equation of state data are also shown (x) (7). Points (+) A and (+) B show the upper and lower limits to the data calibration for shot 1038.

region, would send a finite rarefaction wave propagating back toward the impact plane. The particle velocity increase from the rarefaction wave would be added to the particle velocity from the original shock wave, leading to a short increase in particle velocity recorded in gauges closer to the impact plane. This phenomenon is traced with the dashed lines in Fig. 4.

If the region of decreased porosity were a vacuum gap then the rarefaction wave should have a similar velocity as the release wave from the back of the flyer plate. The flyer plate release wave can be seen clearly in gauges 1 and 3 at 6.38 and $7.8 \mu \mathrm{s}$, respectively. In gauge 2 , the flyer release wave is overlain with the rarefaction from gauge 3 propagating back toward gauge 2 . The release wave arrives at gauge 4 just before the shock wave. The peak particle velocity and the slope of the rise of the wave in gauge 4 are less than the first 3 gauges. The velocity of the flyer release wave, based on the arrival at gauges 1 and 3 and the compressed distance between the two gauges, is $1.84 \mathrm{~km} / \mathrm{s}$.

The time of arrival of the rarefaction waves from the increased porosity region around each gauge is similar to the flyer release wave veloc- 


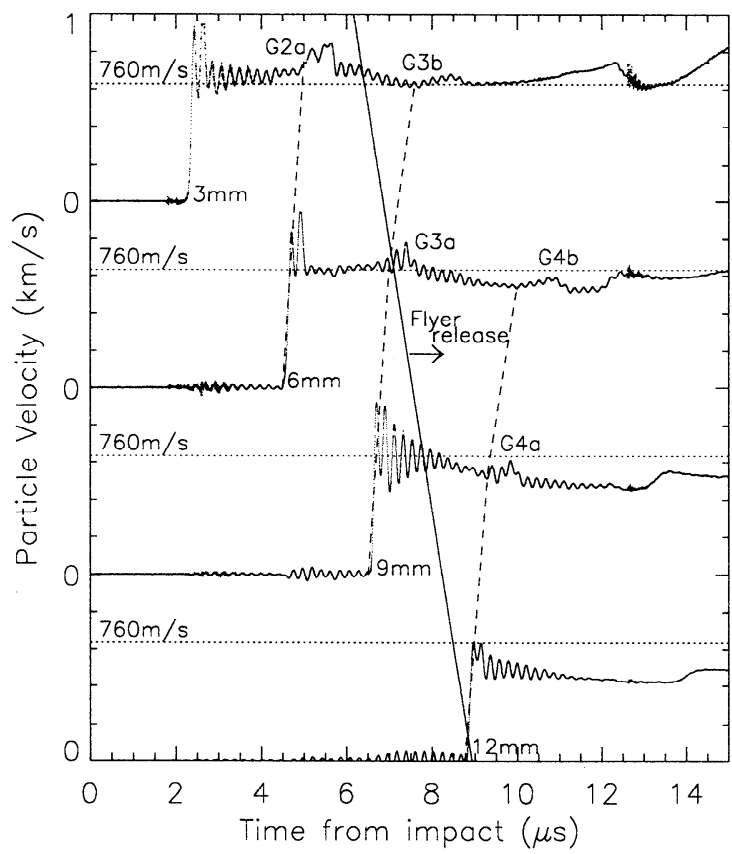

Figure 4: Release and rarefaction waves. See discussion in text.

ity. The timing of these rarefaction waves may be derived from gauges 1 and 2 using features G2a and G3a in Fig. 4. The timing of these waves through the compressed ice imply a velocity of 2.62 and $2.66 \mathrm{~km} / \mathrm{s}$ to rcach G2a and G3a respcctively. This velocity is the sum of the rarefaction wave velocity and the particle velocity from the impact shock wave, $0.76 \mathrm{~km} / \mathrm{s}$. So the rarefaction wave velocities are 1.86 and $1.90 \mathrm{~km} / \mathrm{s}$, consistent with the flyer plate release wave velocity.

The rarefaction waves from the region around the gauges continue to propagate toward the impact plane, slowing with distance. The rarefaction wave from gauge 3 is also visible in the gauge 1 data (G3b), and the gauge 4 rarefaction is visible in the gauge 2 data (G4b). The travel time has increased from a combined effect of the dispersion of the rarefaction wave and the release wave from the flyer plate.

The regions of increased porosity around the gauges may be from poor contact between the ice discs. Ice does not easily anneal to the Kapton coating on the velocity gauges. Also, the man- ner in which the ice discs were made, by pressing the ice grains together, leaves each disc with a bottom and top layer of increased porosity whose thickness is on the order of the grain size (180$355 \mu \mathrm{m})$. Thus when the discs are stacked with the velocity gauges forming a boundary between each disc, there remains the region of increased porosity.

The relatively long duration of the increase in particle velocity at the arrival of the shock wave and gauge rarefaction wave features are not consistent with wave reflection due to impedence mismatch between the ice and the gauges. The gauges are too thin to cause features of the time duration observed here.

Detailed modelling of these gauge profiles is in progress. Future experiments will include unsupported shock waves to study shock wave attenuation in both uniaxial strain and spherical wave decay under a range of porosities.

\section{CONCLUSIONS}

We are conducting shock wave propagation experiments into ice of different porositics. Wc have confirmed that our methods may accurately measure the equation of state of porous ice. The equation of state obtained under low temerature and low pressure agrees well with experiments under terrestrial conditions implying that data on terrestrial snow may be applicable to the outer solar system.

\section{ACKNOWLEDGEMENTS}

This work was supported under NASA grant NAG53644. Contribution \#8680, Division of Geological and Planetary Sciences. Thanks to C. Liu for advice and many useful discussions. Thanks to $\mathrm{M}$. Long and $\mathrm{P}$. Gelle for their excellent support.

\section{REFERENCES}

1. Veverka J. et al., Science 278, 2109-2114 (1997).

2. Yeomans, D.K. et al., Science 278, 2106-2109 (1997). 3. Housen, K.R. and Holsapple, K.A., Lun. Pl. Sci. Conf. XXX, \#1228 (1999). 4. Jewitt, D., Annu. Rev. Earth Planet. Sci. 27, 287-312 (1999). 5. Davis, D.R. and Farinella, P., Icarus 125, 50-60 (1997). 6. Stewart, S.T. and Ahrens, T.J., Lun. Pl. Sci. Conf. XXX, \#2020 (1999). 7. Furnish, M.D. and Boslough M.B., Sandia Report SAND92-0985 (1996). 8. Seaman, L., J. Appl. Phys. 45, 4303-4314 (1974). 9. Bakanova, A.A. et al., Sov. Phys.-JETP 41, 544-548 (1976). 